\title{
Coupling of electrons to the electromagnetic field in a localized basis
}

\author{
Roland E. Allen* \\ Department of Physics, Texas A\&M University, College Station, Texas 77843, USA
}

(Received 3 June 2008; revised manuscript received 26 July 2008; published 21 August 2008)

\begin{abstract}
A simple formula is obtained for coupling electrons in a complex system to the electromagnetic field. It includes the effect of intra-atomic excitations and nuclear motion, and can be applied in, e.g., first-principlesbased simulations of the coupled dynamics of electrons and nuclei in materials and molecules responding to ultrashort laser pulses. Some additional aspects of nonadiabatic dynamical simulations are also discussed, including the potential of "reduced Ehrenfest" simulations for treating problems where standard Ehrenfest simulations will fail.
\end{abstract}

DOI: 10.1103/PhysRevB.78.064305

PACS number(s): 78.20.Bh, 71.15.Pd

It is now possible to perform first-principles simulations of the coupled dynamics of electrons and nuclei with all nuclear coordinates included ${ }^{1-4}$ rather than a subset of nominal reaction coordinates. For very large systems or when many trajectories are necessary, it is convenient to use a first-principles-based scheme $\mathrm{s}^{5-8}$ with a valence-electron Hamiltonian and ion-ion repulsive potential derived from calculations using density functional or other first-principles techniques. Here we are mainly concerned with the issue of how one can efficiently and accurately couple electrons to the electromagnetic field in such an approach, where matrix elements of various operators between localized basis functions (or "atomic orbitals") can be calculated from first principles, and then used in large-scale calculations for complex systems, such as materials and molecules, responding to applied fields, such as ultrashort laser pulses. ${ }^{9-19}$

Our starting point is, of course, the time-dependent Schrödinger equation,

$$
\begin{gathered}
i \hbar \frac{\partial}{\partial t} \psi(\boldsymbol{x}, t)=\hat{H} \psi(\boldsymbol{x}, t), \\
\hat{H}=\frac{1}{2 m}\left[-i \hbar \nabla-\frac{q}{c} \boldsymbol{A}(\boldsymbol{x}, t)\right]^{2}+U, \quad q=-e .
\end{gathered}
$$

Some time ago, Graf and $\operatorname{Vogl}^{20}$ obtained a result, used in Refs. 13-19, which is the time-dependent version of the Peierls substitution: If $\boldsymbol{H}_{0}$ is the Hamiltonian matrix in a localized basis with no applied field,

$$
H_{0}\left(\ell^{\prime}, \ell\right)=\int d^{3} x \phi_{a^{\prime}}^{*}\left(\boldsymbol{x}-\boldsymbol{X}^{\prime}\right) \hat{H}_{0} \phi_{a}(\boldsymbol{x}-\boldsymbol{X}),
$$

and $\tilde{\boldsymbol{H}}$ is the approximate Hamiltonian when there is an applied field with vector potential $\boldsymbol{A}(\boldsymbol{x}, t)$, then they are related by

$$
\tilde{H}\left(\ell^{\prime}, \ell\right)=H_{0}\left(\ell^{\prime}, \ell\right) e^{i q \bar{A}(t) \cdot\left(X^{\prime}-X\right) / \hbar c},
$$

with

$$
\overline{\boldsymbol{A}}(t)=\left[\boldsymbol{A}\left(\boldsymbol{X}^{\prime}, t\right)+\boldsymbol{A}(\boldsymbol{X}, t)\right] / 2 .
$$

Here $\ell$ labels a localized basis function centered on a nucleus whose instantaneous position is $\boldsymbol{X}(\ell, t)$, and we adopt the convention of normally suppressing the indices $\ell$ and $\ell^{\prime}$ as well as the time $t$ by just writing $\boldsymbol{X}$ and $\boldsymbol{X}^{\prime}$. We will ignore any applied scalar potential $A_{0}$, any $\boldsymbol{\mu}_{B} \cdot \boldsymbol{B}$ spin interactions, and the coupling of ion cores or nuclei to the applied fields since these effects can be easily included when necessary.

With the prescription of Eq. (4), one does not need any new parameters in a calculation that employs either a semiempirical $^{13,14}$ or a first-principles-based ${ }^{15-19}$ Hamiltonian $\boldsymbol{H}_{0}$ whose elements are known as a function of $\left(\boldsymbol{X}-\boldsymbol{X}^{\prime}\right)$. On the other hand, this prescription is in one respect a rather crude approximation: It omits intra-atomic excitations and would therefore give no excitation at all for isolated atoms.

Here a more general version of the result of Ref. 20 will be obtained in a form that is almost equally convenient for large-scale applications although it does require additional parameters-namely dipole matrix elements,

$$
\boldsymbol{\mu}_{0}\left(\ell^{\prime}, \ell\right)=q \int d^{3} x \phi_{a^{\prime}}^{*}\left(\boldsymbol{x}-\boldsymbol{X}^{\prime}\right)(\boldsymbol{x}-\boldsymbol{X}) \phi_{a}(\boldsymbol{x}-\boldsymbol{X}),
$$

and on-site $\left(\boldsymbol{X}^{\prime}=\boldsymbol{X}\right)$ matrix elements of the momentum operator,

$$
\boldsymbol{p}_{0}\left(\ell^{\prime}, \ell\right)=\int d^{3} x \phi_{a^{\prime}}^{*}\left(\boldsymbol{x}-\boldsymbol{X}^{\prime}\right)(-i \hbar \nabla) \phi_{a}(\boldsymbol{x}-\boldsymbol{X}),
$$

where $a$ labels an orbital centered on the nucleus whose instantaneous position is $\boldsymbol{X}$. Recall that $\ell$ labels both nucleus and orbital, so at a given instant in time

$$
\ell \leftrightarrow X, a .
$$

One key step is to expand $\psi$ in terms of London orbitals, which we define to be any localized basis functions $\widetilde{\phi}_{a}$ that are defined in terms of field-independent basis functions $\phi_{a}$ by

$$
\widetilde{\boldsymbol{\phi}}_{a}(\boldsymbol{x}-\boldsymbol{X}, t)=e^{i q \boldsymbol{A}(\boldsymbol{x}, t) \cdot(\boldsymbol{x}-\boldsymbol{X}) / \hbar c} \phi_{a}(\boldsymbol{x}-\boldsymbol{X}) .
$$

Notice that $\widetilde{\phi}_{a}(\boldsymbol{x}-\boldsymbol{X}, t)=\phi_{a}(\boldsymbol{x}-\boldsymbol{X})$ when $\boldsymbol{A}=0$ so that after the application of a laser pulse, for example, the London orbitals return to being standard basis functions. The $\phi_{a}$ need not be a complete set but should, of course, be a large enough set to model all physically relevant phenomena. The relatively weak time dependence of the nuclear positions $\boldsymbol{X}$ is ignored for the moment but will be included below. The 
original Hamiltonian of Eq. (2) can be rewritten as ${ }^{20,21}$

$$
\begin{gathered}
\hat{H}=e^{i q \int \boldsymbol{A}(\boldsymbol{x}, t) \cdot d \boldsymbol{x} / \hbar c} \hat{H}_{0} e^{-i q \int \boldsymbol{A}(\boldsymbol{x}, t) \cdot d \boldsymbol{x} / \hbar c}, \\
\hat{H}_{0}=\hat{\boldsymbol{p}}^{2} / 2 m+U, \quad \hat{\boldsymbol{p}}=-i \hbar \boldsymbol{\nabla},
\end{gathered}
$$

since Eqs. (2) and (10) yield the same result when operating on an arbitrary function, and are therefore the same operator. As will be seen immediately below, there are no problems in interpreting the integral of Eq. (10) in the way that it is used here since it is well defined locally in evaluating each matrix element.

We now need the single approximation that $\boldsymbol{A}(\boldsymbol{x}, t)$ varies slowly with respect to $\boldsymbol{x}$ over an atomic diameter or bond length so that

$$
\boldsymbol{A}(\boldsymbol{x}, t) \approx \overline{\boldsymbol{A}}(t)
$$

in the matrix elements which involve $\phi_{a^{\prime}}\left(\boldsymbol{x}-\boldsymbol{X}^{\prime}\right)$ and $\phi_{a}(\boldsymbol{x}-\boldsymbol{X})$. (The wavelength is thus assumed to be large compared to 1 Å.) When Eqs. (10) and

$$
\psi(\boldsymbol{x}, t)=\sum_{\ell} \psi(\ell, t) \widetilde{\phi}_{a}(\boldsymbol{x}-\boldsymbol{X}, t)
$$

are substituted into Eq. (1), and the resulting equation is subjected to $\int d^{3} x \widetilde{\boldsymbol{\phi}}_{a^{\prime}}^{*}\left(\boldsymbol{x}-\boldsymbol{X}^{\prime}, t\right)$, we then obtain

$$
\sum_{\ell} S\left(\ell^{\prime}, \ell\right) i \hbar \frac{\partial \psi(\ell, t)}{\partial t}=\sum_{\ell} H\left(\ell^{\prime}, \ell\right) \psi(\ell, t),
$$

where

$$
\begin{gathered}
S\left(\ell^{\prime}, \ell\right)=S_{0}\left(\ell^{\prime}, \ell\right) e^{i q \overline{\boldsymbol{A}}(t) \cdot\left(\boldsymbol{X}^{\prime}-X\right) / \hbar c}, \\
S_{0}\left(\ell^{\prime}, \ell\right)=\int d^{3} x \phi_{a^{\prime}}^{*}\left(\boldsymbol{x}-\boldsymbol{X}^{\prime}\right) \phi_{a}(\boldsymbol{x}-\boldsymbol{X}), \\
H\left(\ell^{\prime}, \ell\right)=\tilde{H}\left(\ell^{\prime}, \ell\right)-\overline{\boldsymbol{E}}(t) \cdot \boldsymbol{\mu}\left(\ell^{\prime}, \ell\right), \\
\boldsymbol{\mu}\left(\ell^{\prime}, \ell\right)=\boldsymbol{\mu}_{0}\left(\ell^{\prime}, \ell\right) e^{i q \bar{A}(t) \cdot\left(X^{\prime}-X\right) / \hbar c},
\end{gathered}
$$

and

$$
\overline{\boldsymbol{E}}(t)=-\frac{1}{c} \frac{\partial \overline{\boldsymbol{A}}(t)}{\partial t}
$$

is the electric field. In matrix form, Eq. (14) is

$$
i \hbar \frac{\partial}{\partial t} \psi(t)=\boldsymbol{S}^{-1} \cdot \boldsymbol{H} \cdot \psi(t) .
$$

If there are $N_{e}$ electronic basis functions, then $\psi$ is an $N_{e}$-dimensional vector whereas $\boldsymbol{x}, \boldsymbol{A}, \boldsymbol{\mu}$, etc. are threedimensional vectors. The dipole matrix elements can in principle be obtained in $a b$ initio calculations such as those used to obtain, e.g., the Hamiltonian matrix elements $H_{0}\left(\ell^{\prime}, \ell\right){ }^{5-8}$ Alternatively, one might make the approximation of including only the terms with single-atom dipole matrix elements, $\boldsymbol{\mu}_{0}\left(\boldsymbol{X} a^{\prime}, \boldsymbol{X} a\right)$, and then take these from either atomic calculations or experiment.

We now return to the time dependence of the nuclear positions $\boldsymbol{X}$. With Eq. (13) rewritten as

$$
\psi(x, t)=\sum_{\ell} \tilde{\psi}(\ell, t) \phi_{a}(\boldsymbol{x}-\boldsymbol{X}),
$$

$$
\widetilde{\psi}(\ell, t)=\psi(\ell, t) e^{i q A(x, t) \cdot(x-X) / \hbar c},
$$

we have $\mathrm{e}^{22}$

$$
\frac{\partial \psi}{\partial t}=\sum_{\ell}\left[\frac{\partial \widetilde{\psi}(\ell)}{\partial t} \phi_{a}(\boldsymbol{x}-\boldsymbol{X})+\widetilde{\psi}(\ell) \frac{\partial \phi_{a}(\boldsymbol{x}-\boldsymbol{X})}{\partial \boldsymbol{X}} \cdot \dot{\boldsymbol{X}}\right] .
$$

In order to treat the second term above, we assume (as indicated by the notation) that the basis functions depend only on $(\boldsymbol{x}-\boldsymbol{X})$ so that

$$
\begin{gathered}
\frac{\partial \phi_{a}(\boldsymbol{x}-\boldsymbol{X})}{\partial \boldsymbol{X}}=-\frac{\partial \phi_{a}(\boldsymbol{x}-\boldsymbol{X})}{\partial(\boldsymbol{x}-\boldsymbol{X})}, \\
=-\boldsymbol{\nabla} \phi_{a}(\boldsymbol{x}-\boldsymbol{X}) .
\end{gathered}
$$

There is an additional correction involving $\dot{\boldsymbol{X}}$ that arises from

$$
\begin{aligned}
\frac{\partial \tilde{\psi}(\ell)}{\partial t}= & e^{i q \boldsymbol{A}(\boldsymbol{x}) \cdot(\boldsymbol{x}-\boldsymbol{X}) / \hbar c}\left\{\frac{\partial \psi(\ell)}{\partial t}+\psi(\ell)\left(\frac{i q}{\hbar c}\right)\right. \\
& \left.\times\left[\frac{\partial \boldsymbol{A}(\boldsymbol{x})}{\partial t} \cdot(\boldsymbol{x}-\boldsymbol{X})-\boldsymbol{A}(\boldsymbol{x}) \cdot \dot{\boldsymbol{X}}\right]\right\} .
\end{aligned}
$$

It follows that Eq. (17) is modified to

$$
\begin{aligned}
H\left(\ell^{\prime}, \ell\right)= & H_{0}\left(\ell^{\prime}, \ell\right) e^{i q \bar{A} \cdot\left(\boldsymbol{X}^{\prime}-X\right) / \hbar c}-\overline{\boldsymbol{E}}(t) \cdot \boldsymbol{\mu}\left(\ell^{\prime}, \ell\right) \\
& -\dot{\boldsymbol{X}} \cdot \boldsymbol{P}\left(\ell^{\prime}, \ell\right),
\end{aligned}
$$

where

$$
\begin{gathered}
\boldsymbol{P}\left(\ell^{\prime}, \ell\right)=\boldsymbol{p}\left(\ell^{\prime}, \ell\right)+(q / c) \overline{\boldsymbol{A}} S\left(\ell^{\prime}, \ell\right), \\
\boldsymbol{p}\left(\ell^{\prime}, \ell\right)=\boldsymbol{p}_{0}\left(\ell^{\prime}, \ell\right) e^{i q \overline{\boldsymbol{A}} \cdot\left(\boldsymbol{X}^{\prime}-X\right) / \hbar c},
\end{gathered}
$$

so another set of parameters is needed to treat the time dependence of the basis functions that arises from nuclear motion-namely, the matrix elements of the momentum operator $-i \hbar \nabla$.

However, when $\boldsymbol{X}^{\prime} \neq \boldsymbol{X}$, there is a more convenient way of writing $\boldsymbol{p}_{0}\left(\ell^{\prime}, \ell\right)$ :

$$
\begin{gathered}
\boldsymbol{p}_{0}\left(\ell^{\prime}, \ell\right)=i \hbar \int d^{3} x \phi_{a^{\prime}}^{*}\left(\boldsymbol{x}-\boldsymbol{X}^{\prime}\right) \frac{\partial \phi_{a}(\boldsymbol{x}-\boldsymbol{X})}{\partial \boldsymbol{X}}, \\
=i \hbar \frac{\partial}{\partial \boldsymbol{X}} S_{0}\left(\ell^{\prime}, \ell\right) \quad \text { if } \quad \boldsymbol{X}^{\prime} \neq \boldsymbol{X} .
\end{gathered}
$$

Furthermore, in the usual case of basis functions ("atomic orbitals") that are either even or odd under inversion through the nucleus, the fact that $(\boldsymbol{x}-\boldsymbol{X})$ and $\boldsymbol{\nabla}=\partial / \partial(\boldsymbol{x}-\boldsymbol{X})$ are odd under inversion (with $\boldsymbol{X}$ here taken to be fixed) implies that

$$
\boldsymbol{\mu}_{0}(\ell, \ell)=\boldsymbol{p}_{0}(\ell, \ell)=0 .
$$

Notice that Eq. (25) respects gauge invariance: If

$$
\overline{\boldsymbol{A}}(t) \rightarrow \overline{\boldsymbol{A}}^{\prime}(t)=\overline{\boldsymbol{A}}(t)+\Delta \overline{\boldsymbol{A}},
$$

where $\Delta \overline{\boldsymbol{A}}$ is independent of $t$, then Eq. (14) still holds with 


$$
\psi(\ell, t) \rightarrow \psi^{\prime}(\ell, t)=e^{i q \Delta \bar{A} \cdot X / \hbar c} \psi(\ell, t) .
$$

This is the discrete version of

$$
\begin{gathered}
\boldsymbol{A}(\boldsymbol{x}, t) \rightarrow \boldsymbol{A}^{\prime}(\boldsymbol{x}, t)=\boldsymbol{A}(\boldsymbol{x}, t)+\nabla \Lambda(\boldsymbol{x}), \\
\psi(\boldsymbol{x}, t) \rightarrow \psi^{\prime}(\boldsymbol{x}, t)=e^{i q \Lambda(\boldsymbol{x}) / \hbar c} \psi(\boldsymbol{x}, t) .
\end{gathered}
$$

If $\Delta \overline{\boldsymbol{A}}$ is a function of $t$, gauge invariance again holds but with the scalar potential included.

Equation (25) is the central result of the present note. This effective Hamiltonian is not manifestly Hermitian but it still conserves probability and preserves the Pauli principle since a straightforward calculation using Eq. (25) in Eq. (14) gives

$$
i \hbar \partial\left(\psi_{n^{\prime}}^{\dagger} \cdot \boldsymbol{S} \cdot \psi_{n}\right) / \partial t=0
$$

where $n$ labels a time-dependent one-electron state. This result also follows from the original Schrödinger equation [Eq. (1)] and the expansion of Eq. (13) since

$$
\int d^{3} x \psi_{n^{\prime}}^{*}(\boldsymbol{x}, t) \psi_{n}(\boldsymbol{x}, t)=\psi_{n^{\prime}}^{\dagger}(t) \cdot \boldsymbol{S}(t) \cdot \psi_{n}(t),
$$

but it is reassuring that the approximation of Eq. (12) preserves orthonormality of the time-dependent states.

For slowly moving nuclei, the last term in Eq. (25) is not important. (It may be worth mentioning in this context that the direct coupling of the nuclei to the field is not considered here since it can be treated separately.) In an earlier paper ${ }^{23}$ we argued that the nuclear motion can be approximately treated as a "nuclear velocity field" analogous to the radiation field and, in this spirit, we obtained (as a crude approximation) a generalized Peierls substitution:

$$
\begin{aligned}
H_{\mathrm{eff}}\left(\ell^{\prime}, \ell\right)= & e^{(i / \hbar)\left[(q / c) \boldsymbol{A}\left(\boldsymbol{X}^{\prime}\right)+m \dot{\boldsymbol{X}}^{\prime}\right] \cdot \boldsymbol{X}^{\prime}} \\
& \times H_{0}\left(\ell^{\prime}, \ell\right) e^{-(i / \hbar)[(q / c) \boldsymbol{A}(\boldsymbol{X})+m \dot{\boldsymbol{X}}] \cdot \boldsymbol{X}} .
\end{aligned}
$$

We also used this modified Hamiltonian in calculations for organic molecules responding to femtosecond-scale laser pulses of moderately strong intensity $\left(\sim 10^{12} \mathrm{~W} / \mathrm{cm}^{2}\right)$ and found that the $\dot{X}$ terms made very little difference in the final results. On the other hand, the two-center momentum matrix elements can be obtained from Eq. (29) and the nonzero one-center matrix elements from either atomic calculations or experiment, so it is certainly feasible to include the last term in Eq. (25). Notice that this term is different from the Pulay correction, ${ }^{1}$ which also results from the fact that the basis functions follow the nuclei but occurs in the equation of motion for the nuclei rather than the time-dependent Schrödinger equation for the electrons. In the kind of approach considered here, there is no Pulay correction because the Hamiltonian matrix elements are supposed to have a position dependence that includes the movement of the basis functions.

In this context, it is worth noting that the "Ehrenfest dynamics" 24,25 of, e.g., time-dependent density-functional theory (TDDFT) and the density-functional-based calculations of Refs. 15-19, can be substantially improved in molecular calculations via a trivially different procedure that might be called "reduced Ehrenfest dynamics" and which is similar in spirit to the surface hopping methods of Tully ${ }^{26}$ and others. ${ }^{27}$ Let us first recall some well-known results: The total wave function for a system of nuclei with coordinates $X_{n}$ and electrons with coordinates $x_{e}$ can be represented by the Born-Oppenheimer expansion:

$$
\Psi^{\mathrm{tot}}\left(X_{n}, x_{e}, t\right)=\sum_{i} \Phi_{i}\left(X_{n}, t\right) \Psi_{i}\left(x_{e}, X_{n}\right) .
$$

The basis functions $\Psi_{i}$ are the electronic eigenstates at fixed $X_{n}$ with the electron-nuclei and nuclei-nuclei interactions included in the electronic Hamiltonian $H_{e}$ :

$$
H_{e}\left(X_{n}\right) \Psi_{i}\left(x_{e}, X_{n}\right)=E_{i}\left(X_{n}\right) \Psi_{i}\left(x_{e}, X_{n}\right) .
$$

Substitution into the Schrödinger equation,

$$
i \hbar \partial \Psi^{\text {tot }} / \partial t=\mathcal{H} \Psi^{\text {tot }}, \quad \mathcal{H}=T_{n}+H_{e},
$$

where $T_{n}$ is the nuclear kinetic-energy operator, gives an equation of the form ${ }^{28,29}$

$$
\begin{gathered}
i \hbar \frac{\partial}{\partial t} \Phi_{i}=\left(T_{n}+E_{i}\right) \Phi_{i}-\sum_{j} \Lambda_{i j} \Phi_{j}, \\
\Lambda_{i j}=\frac{\hbar^{2}}{2 M_{n}}\left(2 \boldsymbol{F}_{i j} \cdot \nabla_{n}+G_{i j}\right), \\
\boldsymbol{F}_{i j}=\left\langle i\left|\nabla_{n}\right| j\right\rangle, \quad G_{i j}=\left\langle i\left|\nabla_{n}^{2}\right| j\right\rangle,
\end{gathered}
$$

where $M_{n}$ is a representative nuclear mass and $\boldsymbol{\nabla}_{n}$ involves all the appropriately rescaled nuclear coordinates. If there are $N_{n}$ relevant nuclear coordinates, then $\boldsymbol{\nabla}_{n}$ and $\boldsymbol{F}$ are $N_{n}$-dimensional vectors. Also, quantities in the last line are matrix elements defined in terms of $\Psi_{i}^{\dagger}$ and $\Psi_{j}$ in the usual way. If the components $\Phi_{i}$ are assembled into a vector $\Phi$, Eq. (41) can be written in a form that resembles a nonabelian gauge theory: ${ }^{30}$

$$
i \hbar \frac{\partial}{\partial t} \Phi=\left[-\frac{\hbar^{2}}{2 M_{n}}\left(\boldsymbol{\nabla}_{n}+\boldsymbol{F}\right)^{2}+\boldsymbol{E}\right] \cdot \Phi,
$$

where $\boldsymbol{E}$ is the diagonal matrix with elements $E_{i}$. Finally, it can be shown that ${ }^{31}$

$$
\boldsymbol{F}_{i j}=\frac{\left\langle i\left|\nabla_{n} H_{e}\right| j\right\rangle}{E_{j}-E_{i}}, \quad E_{i} \neq E_{j} .
$$

This last equation implies that each term in the BornOppenheimer expansion should evolve nearly independently if it is sufficiently distant in energy from all the other terms: If

$$
\left|E_{i}-E_{j}\right| \gg\left|\left\langle i\left|\nabla_{n} H_{e}\right| j\right\rangle\right|\left(\hbar / P_{i}\right),
$$

where $P_{i}=\left(2 M_{n} E_{i}\right)^{1 / 2}$, then

$$
i \hbar \partial \Phi_{i} / \partial t \approx\left(T_{n}+E_{i}\right) \Phi_{i} .
$$

This is the time-dependent Born-Oppenheimer or adiabatic approximation.

On the other hand, whenever nuclear motion causes two Born-Oppenheimer "potential-energy surfaces" to approach each other, so that 


$$
\left|E_{i}-E_{j}\right| \lesssim\left|\left\langle i\left|\nabla_{n} H_{e}\right| j\right\rangle\right|\left(\hbar / P_{i}\right),
$$

there is a nonadiabatic interaction ${ }^{28,29,32,33}$ and a BornOppenheimer simulation based on Eq. (47) is invalid.

The results of Refs. 15-19 have provided a clear demonstration of the following features of simulations based on Ehrenfest dynamics: (1) Electronic transitions are automatically observed at the points of closest approach where Eq. (48) holds, with energy released to molecular vibrations. These points are, of course, avoided crossings near the conical intersections in configuration space predicted by Teller. ${ }^{28,29,34}$ (2) These transitions occur rapidly over a time interval of $\sim 1 \mathrm{fs}$, during which the nuclei do not move appreciably.

Ehrenfest simulations are based on the equation of motion for the Heisenberg operator $\hat{X}(t)$ representing any nuclear coordinate: $:^{24,25}$

$$
M d^{2} \hat{X} / d t^{2}=-\partial \mathcal{H} / \partial \hat{X}
$$

Here $M$ is the corresponding nuclear mass and $\mathcal{H}$ is the Hamiltonian of the system. In a standard Ehrenfest simulation, the expectation value is taken over the full state of the system, including excitations (e.g., by a laser pulse) and deexcitations (e.g., by nuclear motion near conical intersections):

$$
M \frac{d^{2}\langle\hat{X}\rangle}{d t^{2}}=-\left\langle\frac{\partial \mathcal{H}(\hat{X})}{\partial \hat{X}}\right\rangle \approx-\frac{\partial \mathcal{H}(\langle\hat{X}\rangle)}{\partial\langle\hat{X}\rangle} .
$$

There are clearly two weaknesses with this approach: First, the equality on the left represents an average over all the terms in the expansion of Eq. (38) with each term representing a different nuclear trajectory. Second, the approximation on the right is totally invalid if these trajectories are very different.

Suppose that, however, the standard procedure for an Ehrenfest simulation is replaced by a trivially different procedure in which the state of the system is collapsed to a single Born-Oppenheimer term immediately after an excitation or deexcitation event. Then Eq. (47) implies that it will essentially remain in this single adiabatically evolving state until the next such event. For this reduced electronic state, the nuclei will ordinarily follow a single trajectory except for quantum fluctuations of order $\left\langle(\hat{X}-\langle\hat{X}\rangle)^{2}\right\rangle{ }^{35}$ It is still possible for nuclear wave packets to diverge on a single potential-energy surface, but one does not expect this to be a common occurrence for processes in which the most relevant nuclei are reasonably heavy.

For simplicity, first consider a very short laser pulse (e.g., $\sim 1-5$ fs in duration) applied to a molecule. The procedure for a reduced Ehrenfest simulation is as follows: Start with a single electronic eigenstate (e.g., the ground state) and initially perform an Ehrenfest simulation in the usual way. Immediately following the pulse, the molecule will be in a superposition of electronic eigenstates:

$$
\Psi_{e}(t)=\sum_{i} c_{i} \Psi_{i}
$$

At this point one collapses $\Psi_{e}$ to a single eigenstate $\Psi_{i}$ and continues the simulation, with $\langle\hat{X}\rangle$ now interpreted as the expectation value for this single resulting time-dependent state, until another significant excitation or deexcitation is observed, after which there is again a further reduction to a single electronic eigenstate.

There are potentially a substantial number of branches to be followed during this sort of simulation, corresponding to the various states in the superposition of Eq. (51) after an excitation or deexcitation event. The goal, however, is to understand the most relevant processes and there will ordinarily be physical motivations for selecting the most interesting branches. Similarly, there will be many branches emerging during an excitation process whose duration is long enough for the nuclei to move appreciably before it is completed (e.g., a femtosecond-scale laser pulse whose duration is still $\gg 1 \mathrm{fs}$ ) and a choice among the branches again has to be based on physical considerations.

For a molecule subjected to high-frequency or highintensity radiation, the branches include ionized states. The one-electron matrix element between an orbital $\ell$ and an ionized state with momentum $\boldsymbol{p}$ is

$$
H_{\ell p}=\frac{e}{m c} \boldsymbol{A}(\boldsymbol{X}, t) \cdot\langle\ell|\hat{\boldsymbol{p}}| \boldsymbol{p}\rangle .
$$

For a crude description of ionization, one might add a model orbital $\phi_{0}$ to the basis with

$$
H_{0 \ell}=\alpha_{\ell} \frac{e}{m c}|\boldsymbol{A}(\boldsymbol{X}, t)| p_{0}, \quad H_{\ell 0}=0,
$$

where $p_{0} \sim \hbar / a_{0}, a_{0}$ is the Bohr radius, and $\alpha_{\ell}$ is an adjustable dimensionless parameter. This non-Hermitian Hamiltonian removes amplitude from the orbital $\ell$ at each time step and does not return it, so it crudely models excitation to a localized wave packet with the electron ultimately escaping the system. An appreciable probability for a given ionized state then provides motivation for following that branch in a reduced Ehrenfest simulation. Notice that an accurate treatment of ionization is not necessary if the only issue is whether an ionized state is important enough to warrant a simulation of the subsequent dynamics in that state. Also notice that the energy $H_{00}$ of the extra orbital is irrelevant (so one can take $H_{00}=0$ ) and that a single extra orbital is sufficient regardless of the size of the system.

After each wave-function collapse, the use of Eq. (50) implies that the nuclei are treated classically. It is then appropriate to use the mixed classical-quantum action ${ }^{25,36} \mathrm{~S}$ $=\int d t L$, where

$$
L=\frac{1}{2}\left\langle\Psi_{e}\left|\left(i \hbar \frac{\partial}{\partial t}-\mathcal{H}_{e}\right)\right| \Psi_{e}\right\rangle+\text { h.c. }+\frac{1}{2} \sum_{k \alpha} M_{k}\left(\frac{d X_{k \alpha}}{d t}\right)^{2}-U_{\text {rep }},
$$

where $\mathcal{H}_{e}$ is the electronic Hamiltonian, $\left|\Psi_{e}\right\rangle$ is the electronic state, "h.c." means "Hermitian conjugate," $k$ labels a nucleus 
with spatial coordinates $\alpha$, and $U_{\text {rep }}$ is the repulsive interaction between nuclei or ion cores. As shown in Ref. 36 [but with $\boldsymbol{H}$ now given by Eq. (25)], extremalization of this action leads to Eq. (20) and

$$
M \frac{d^{2} X}{d t^{2}}=-\frac{1}{2} \sum_{n} \psi_{n}^{\dagger} \cdot\left(\frac{\partial \boldsymbol{H}}{\partial X}-i \hbar \frac{\partial \boldsymbol{S}}{\partial X} \frac{\partial}{\partial t}\right) \cdot \psi_{n}+\text { h.c. }-\frac{\partial U_{\mathrm{rep}}}{\partial X},
$$

if one makes the usual time-dependent effective-field approximation with exchange and correlation represented by an effective one-electron potential, and the electronic state represented by a single antisymmetrized product wave function $\Psi_{e}(t)$. Here $X$ is any nuclear coordinate and $M$ is the corresponding mass.

The reduced Ehrenfest method described above combines the advantages of Born-Oppenheimer simulations, which are valid when Eq. (47) holds, and Ehrenfest simulations, which are suitable for treating the vibronic transitions when Eq. (48) holds, as the results of Refs. 15-19 have clearly demonstrated. The use of reduced Ehrenfest simulations should solve various problems that are encountered in standard Ehrenfest simulations-for example, the apparent failure of TDDFT to correctly describe the isomerization of retinal. ${ }^{8}$ One problem with TDDFT is that the energies of excited states are not accurately described. However a potentially more severe problem in the case of molecules is that TDDFT is a special case of standard Ehrenfest dynamics and, as a result, fails to yield a complete return to the ground state following deexcitation near a conical intersection. In a reduced Ehrenfest simulation, on the other hand, one correctly follows the nuclear dynamics for that fraction of the population of molecules that does return to the ground state, and which therefore should isomerize more readily. Reduced Ehrenfest simulations are practical for large molecules and are still consistent with the true meaning of quantum amplitudes, which yield probabilities for the various outcomes that are observed at the classical level.

Finally, it may be worth noting that the above treatment can be straightforwardly generalized to other particles, relativistic systems, and nonabelian gauge fields, with $\psi$ in Eq. (1) interpreted as a multicomponent field and the Hamiltonian of Eq. (2) appropriately changed. It can also be used with many-body effects included through self-energy terms, in the Kadanoff-Baym/Keldysh equations for time-dependent and nonequilibrium problems. ${ }^{37,38}$ The chief limitation is the use of localized basis functions and the approximation of Eq. (12).

This work was supported by the Robert A. Welch Foundation (Grant No. A-0929). The author also thanks Yusheng Dou and Meng Gao for their helpful comments. *allen@tamu.edu

${ }^{1}$ M. C. Payne, M. P. Teter, D. C. Allan, T. A. Arias, and J. D. Joannopoulos, Rev. Mod. Phys. 64, 1045 (1992).

${ }^{2}$ J. Li, G. Speyer, and O. F. Sankey, Phys. Rev. Lett. 93, 248302 (2004).

${ }^{3}$ J. Van Heys, M. Lindenblatt, and E. Pehlke, Phase Transitions 78, 773 (2005).

${ }^{4}$ Time-Dependent Density Functional Theory, edited by M. A. L. Marques, C. A. Ullrich, F. Nogueira, A. Rubio, K. Burke, and E. K. U. Gross (Springer, Heidelberg, 2006).

${ }^{5}$ D. Porezag, Th. Frauenheim, Th. Köhler, G. Seifert, and R. Kaschner, Phys. Rev. B 51, 12947 (1995).

${ }^{6}$ M. Elstner, D. Porezag, G. Jungnickel, J. Elsner, M. Haugk, Th. Frauenheim, S. Suhai, and G. Seifert, Phys. Rev. B 58, 7260 (1998).

${ }^{7}$ Th. Frauenheim, G. Seifert, M. Elstner, T. Niehaus, C. Köhler, M. Amkreutz, M. Sternberg, Z. Hajnal, A. D. Carlo, and S. Suhai, J. Phys.: Condens. Matter 14, 3015 (2002).

${ }^{8}$ T. A. Niehaus, D. Heringer, B. Torralva, and Th. Frauenheim, Eur. Phys. J. D 35, 467 (2005).

${ }^{9}$ S. K. Sundaram and E. Mazur, Nat. Mater. 1, 217 (2002).

${ }^{10}$ A. M. Lindenberg, J. Larsson, K. Sokolowski-Tinten, K. J. Gaffney, C. Blome, O. Synnergren, J. Sheppard, C. Caleman, A. G. MacPhee, D. Weinstein, D. P. Lowney, T. K. Allison, T. Matthews, R. W. Falcone, A. L. Cavalieri, D. M. Fritz, S. H. Lee, P. H. Bucksbaum, D. A. Reis, J. Rudati, P. H. Fuoss, C. C. Kao, D. P. Siddons, R. Pahl, J. Als-Nielsen, S. Duesterer, R. Ischebeck, H. Schlarb, H. Schulte-Schrepping, Th. Tschentscher, J. Schneider, D. von der Linde, O. Hignette, F. Sette, H. N. Chap- man, R. W. Lee, T. N. Hansen, S. Techert, J. S. Wark, M. Bergh, G. Huldt, D. van der Spoel, N. Timneanu, J. Hajdu, R. A. Akre, E. Bong, P. Krejcik, J. Arthur, S. Brennan, K. Luening, and J. B. Hastings, Science 308, 392 (2005).

${ }^{11}$ P. B. Hillyard, K. J. Gaffney, A. M. Lindenberg, S. Engemann, R. A. Akre, J. Arthur, C. Blome, P. H. Bucksbaum, A. L. Cavalieri, A. Deb, R. W. Falcone, D. M. Fritz, P. H. Fuoss, J. Hajdu, P. Krejcik, J. Larsson, S. H. Lee, D. A. Meyer, A. J. Nelson, R. Pahl, D. A. Reis, J. Rudati, D. P. Siddons, K. Sokolowski-Tinten, D. von der Linde, and J. B. Hastings, Phys. Rev. Lett. 98, 125501 (2007).

${ }^{12}$ A. H. Zewail, Femtochemistry: Ultrafast Dynamics of the Chemical Bond (World Scientific, Singapore, 1994); A. H. Zewail, Angew. Chem., Int. Ed. 39, 2586 (2000).

${ }^{13}$ J. S. Graves and R. E. Allen, Phys. Rev. B 58, 13627 (1998).

${ }^{14}$ T. Dumitrica and R. E. Allen, Phys. Rev. B 66, 081202(R) (2002).

${ }^{15}$ B. Torralva, T. A. Niehaus, M. Elstner, S. Suhai, Th. Frauenheim, and R. E. Allen, Phys. Rev. B 64, 153105 (2001).

${ }^{16}$ Y. Dou and R. E. Allen, Chem. Phys. Lett. 378, 323 (2003).

${ }^{17}$ Y. Dou, B. R. Torralva, and R. E. Allen, Chem. Phys. Lett. 392, 352 (2004).

${ }^{18}$ P. Sauer and R. E. Allen, Chem. Phys. Lett. 434, 260 (2007).

${ }^{19}$ P. Sauer and R. E. Allen, Chem. Phys. Lett. 450, 192 (2008).

${ }^{20}$ M. Graf and P. Vogl, Phys. Rev. B 51, 4940 (1995).

${ }^{21}$ R. Peierls, Z. Phys. 80, 763 (1933).

${ }^{22}$ T. N. Todorov, J. Phys.: Condens. Matter 13, 10125 (2001).

${ }^{23}$ T. Dumitrica, A. Burzo, Y. Dou, and R. E. Allen, Phys. Status Solidi B 241, 2331 (2004). 
${ }^{24}$ J. J. Sakurai, Modern Quantum Mechanics (Addison-Wesley, Reading, MA, 1994).

${ }^{25}$ R. E. Allen, Phys. Rev. B 50, 18629 (1994).

${ }^{26}$ J. C. Tully, J. Chem. Phys. 93, 1061 (1990).

${ }^{27}$ G. Granucci and M. Persico, J. Chem. Phys. 126, 134114 (2007), and references therein.

${ }^{28}$ Conical Intersections: Electronic Structure, Dynamics, and Spectroscopy, edited by W. Domcke, D. R. Yarkony, and H. Köppel (World Scientific, Singapore, 2004).

${ }^{29}$ M. Baer, Beyond Born-Oppenheimer: Electronic Nonadiabatic Coupling Terms and Conical Intersections (Wiley, Hoboken, 2006).

${ }^{30}$ B. Zygelman, Phys. Lett. A 125, 476 (1987).

${ }^{31}$ S. T. Epstein, Am. J. Phys. 22, 613 (1954).
${ }^{32}$ L. S. Cederbaum, J. Chem. Phys. 128, 124101 (2008), and references therein.

${ }^{33}$ M. Ben-Nun, J. Quenneville, and T. J. Martínez, J. Phys. Chem. A 104, 5161 (2000).

${ }^{34}$ E. Teller, J. Phys. Chem. 41, 109 (1937).

${ }^{35}$ P. Sauer, Y. Rostovtsev, and R. E. Allen, J. Chem. Phys. 126, 024502 (2007).

${ }^{36}$ R. E. Allen, T. Dumitrica, and B. Torralva, Ultrafast Physical Processes in Semiconductors, edited by K. T. Tsen (Academic, New York, 2001), Chap. 7.

${ }^{37}$ Introduction to Computational Methods in Many Body Physics, edited by M. Bonitz and D. Semkat (Rinton, Paramus, NJ, 2006), and references therein.

${ }^{38}$ N. E. Dahlen and R. van Leeuwen, Phys. Rev. Lett. 98, 153004 (2007). 